\title{
Neural correlates of mirth and laughter: a direct electrical cortical stimulation study.
}

\section{$\operatorname{AUTHOR}(\mathrm{S}):$}

Yamao, Yukihiro; Matsumoto, Riki; Kunieda, Takeharu; Shibata, Sumiya; Shimotake, Akihiro; Kikuchi, Takayuki; Satow, Takeshi; ... Fukuyama, Hidenao; Ikeda, Akio; Miyamoto, Susumu

\section{CITATION:}

Yamao, Yukihiro ...[et al]. Neural correlates of mirth and laughter: a direct electrical cortical stimulation study.. Cortex 2015, 66: 134-140

\section{ISSUE DATE:}

2015-05

URL:

http://hdl.handle.net/2433/210373

\section{RIGHT:}

(c) 2015. This manuscript version is made available under the CC-BY-NC-ND 4 O license

http://creativecommons.org/licenses/by-nc-nd/4.0/; The full-text file will be made open to the public on 1 May 2016 in accordance with publisher's 'Terms and Conditions for Self-Archiving'.; This is not the published version. Please cite only the published version.; この論文は出版社版でありません。引用の際には出版社版をご確認ご利用ください。 
Neural correlates of mirth and laughter: a direct electrical cortical stimulation study

Yukihiro Yamao ${ }^{1)}$ Riki Matsumoto ${ }^{2)}$ Takeharu Kunieda $^{1)}$ Sumiya Shibata ${ }^{1)}$

Akihiro Shimotake ${ }^{3)}$ Takayuki Kikuchi ${ }^{1)}$ Takeshi Satow $^{4)}$ Nobuhiro Mikuni ${ }^{5)}$

Hidenao Fukuyama ${ }^{6)}$ Akio Ikeda $^{2)}$ Susumu Miyamoto ${ }^{1)}$

1) Department of Neurosurgery, Kyoto University Graduate School of Medicine

2) Department of Epilepsy, Movement Disorders and Physiology, Kyoto University

Graduate School of Medicine

3) Department of Neurology, Kyoto University Graduate School of Medicine

4) Department of Neurosurgery, Nagahama City Hospital

5) Department of Neurosurgery, Sapporo Medical University School of Medicine

6) Human Brain Research Center, Kyoto University Graduate School of Medicine

Corresponding to: Riki Matsumoto \& Takeharu Kunieda,

Kyoto University Graduate School of Medicine, Kyoto, Japan 
54,Shogoin Kawahara-cho, Sakyo-ku, Kyoto, 606-8507, Japan

tel \& fax: +81-75-751-3772 \& +81-75-751-9416

e-mail: matsumot@kuhp.kyoto-u.ac.jp (RM), kuny@kuhp.kyoto-u.ac.jp (TK)

\section{Highlights:}

1) Direct cortical stimulation revealed a close relationship between mirth and language.

2) When mesial temporal structures were intact, electrical cortical stimulation first elicited contralateral facial movement, followed by bilateral facial movements with mirth.

3) Emotional facial movements had contralateral dominance.

Abbreviations: $\mathrm{ES}$ = electrical cortical stimulation, $\mathrm{fMRI}=$ functional magnetic resonance imaging, $\mathrm{HS}$ = hippocampal sclerosis, $\mathrm{MEP}=$ motor evoked potential, EMG = electromyogram, SMA = supplementary motor area, BTLA = basal temporal language area 


\section{Abstract}

Laughter consists of both motor and emotional aspects. The emotional component, known as mirth, is usually associated with the motor component, namely, bilateral facial movements. Previous electrical cortical stimulation (ES) studies revealed that mirth was associated with the basal temporal cortex, inferior frontal cortex, and medial frontal cortex. Functional neuroimaging implicated a role for the left inferior frontal and bilateral temporal cortices in humor processing. However, the neural origins and pathways linking mirth with facial movements are still unclear. We hereby report two cases with temporal lobe epilepsy undergoing subdural electrode implantation in whom ES of the left basal temporal cortex elicited both mirth and laughter-related facial muscle movements. In one case with normal hippocampus, high-frequency ES consistently caused contralateral facial movement, followed by bilateral facial movements with mirth. In contrast, in another case with hippocampal sclerosis, ES elicited only mirth at low intensity and short duration, and eventually laughter at higher intensity and longer duration. In both cases, the basal temporal language area was 
located within or adjacent to the cortex where ES produced mirth. In conclusion, the present direct ES study demonstrated that 1) mirth had a close relationship with language function, 2) intact mesial temporal structures were actively engaged in the beginning of facial movements associated with mirth, and 3) these emotion-related facial movements had contralateral dominance.

Keywords: mirth; laughter; electrical cortical stimulation; mesial temporal structure;

basal temporal language area 


\section{Introduction}

Laughter, an essential part of daily life, consists of motor and emotional components, the latter of which is known as mirth (Arroyo et al., 1993). Indeed, appreciating or enjoying humor is associated with a feeling of mirth. Lesion studies have shown that humor consists of both cognitive and affective processing (Gardner, Ling, Flamm, \& Silverman, 1975). Goel and Dolan (2001), in their pioneer functional magnetic resonance imaging (fMRI) study, showed that cognitive processing with semantic components was associated with the left hemisphere (left inferior frontal gyrus and posterior inferior temporal gyrus), while cognitive processing with semantic components involved the bilateral temporal cortices (bilateral posterior middle temporal gyrus and left posterior inferior temporal gyrus), and that affective processing was associated with reward processing system (medial ventral prefrontal cortex) (Goel \& Dolan, 2001). This notion was confirmed by several lines of evidence using fMRI in healthy subjects (Amir, Biederman, Wang, \& Xu, 2013; Mobbs, Greicius, Abdel-Azim, Menon, \& Reiss, 2003; Moran, Wig, Adams, Janata, \& Kelley, 2004; Watson, 
Matthews, \& Allman, 2007). However, despite centuries of inquiry, the neural origins and pathways linking bilateral facial movements with mirth are still unclear.

Unilateral lower facial motor weakness (contralesional “mimetic palsy” or emotional facial paresis), which manifests during spontaneous smiling and weeping but not at all during voluntary muscle contraction, has been reported in patients with lesions involving mesial temporal structures (amygdala and hippocampus) (Hopf, Muller-Forell, \& Hopf, 1992). This suggests that impairment of contralateral functional connections originating in mesial temporal structures leads to asymmetric emotional facial movements.

We report two patients with temporal lobe epilepsy who underwent subdural electrode implantation, and in whom electrical cortical stimulation (ES) of the cortices of the left basal temporal lobe elicited mirth, followed by laughter. By analyzing the features common to the two cases, we postulated that mesial temporal structures directly bridged mirth and laughter.

\section{Materials and Methods}




\subsection{Subjects}

We enrolled 13 consecutive patients with medically intractable left temporal lobe

epilepsy who underwent chronic subdural electrode implantation over the basal part of the temporal lobe for presurgical evaluation between March 2000 and December 2013.

All the patients showed language dominance in the left hemisphere as assessed by the Wada test (Takayama et al., 2004), with the exception of one who demonstrated bilateral language representation. We systematically performed high-frequency ES at the basal part of the temporal lobe in order to map the basal temporal language area (BTLA), as this area is actively engaged in semantic language processing and preservation of the BTLA and its white matter connection previously led to preservation or improvement of verbal memory (Mikuni et al., 2006; Shimotake et al., 2014; Usui et al., 2003). In two of the 13 patients, mirth was elicited by high-frequency ES. Both patients had language dominance in the left hemisphere. Postoperative histology showed an intact hippocampus in one patient (Patient 1) and hippocampal sclerosis (HS) in the other (Patient 2). Neither laughter nor mirth occurred during the patients’ habitual seizures, and Patient 2 showed mimetic facial palsy on the right side only when 
smiling and not at all during voluntary upward curving of the lips. The case of Patient 2 was reported preliminarily as a letter (Satow et al., 2003). The demographic details of Patients 1 and 2 are shown in the supplementary material.

\subsection{High-frequency electrical cortical stimulation}

High-frequency ES (50 Hz, square-wave pulse of alternating polarity with a pulse width of $0.3 \mathrm{~ms}, 1-5 \mathrm{sec}, 1-15 \mathrm{~mA}$ ) was applied to the basal temporal cortices through a pair of implanted electrodes to define the functional areas, especially those related to language function (Matsumoto et al., 2011). The amplitude of the electric current was increased gradually until positive motor symptoms (e.g., contraction of arms or facial muscles) or subjective perception of epileptic aura or somatosensory, visual and auditory sensation occurred; patients were instructed, if present, to report all of these after each stimulation trial was over. When the stimulus intensity was increased $>10$ $\mathrm{mA}$ without any positive motor symptoms or subjective perceptions, the absence of positive (e.g., tonic contraction) and negative (e.g., impairment of rapid alternating movements) tongue motor responses was confirmed, and a series of language batteries 
were administered, each lasting 3-5 sec. This language mapping is described in detail elsewhere (Matsumoto et al., 2011; Usui et al., 2003). We judged the induced or impaired behaviors as significant only when the findings were reproducible (at least two trials) without afterdischarges. In cases of frequent afterdischarges, we decreased the stimulation intensity by 1-2 mA so that these did not occur. The majority of high-frequency ES was performed in a bipolar fashion (i.e., stimulation at two adjacent electrodes). In addition, we occasionally performed monopolar stimulation with reference to an electrode in the nonfunctional cortex to further localize the language areas for the clinical purpose.

\subsection{Assessments of mirth and laughter-related facial muscle movements}

Following every ES trial, if the patient spontaneously reported a feeling of mirth, the examiners (Y.Y., T.S.) inquired as to its presence and character. Since mirth is solely subjective, we judged the reported feeling as significant when the findings were reproducible (in at least two trials) without afterdischarges. In Patient 1, we also performed sham stimulation (i.e., stimulation at $0 \mathrm{~mA}$ intensity) to confirm that mirth 
was induced only by real ES. In order to reproducibly characterize mirth and its associated facial movements, additional trials were performed for research purposes.

After the first subjective report of mirth, the stimulation intensity was increased gradually up to $15 \mathrm{~mA}$ in order to assess the language function. This was only possible in Patient 2, since induced mirth and laughter prevented Patient 1 from properly completing the language batteries. This high-frequency ES study was approved by the Ethics Committee, Kyoto University Graduate School of Medicine (No. 79), and both patients gave written informed consent.

In Patient 1, because asymmetric emotional facial movements were clearly observed in early trials of high-frequency ES, surface electromyogram (EMG) electrodes were placed on the bilateral levator labii superior and orbicularis oris muscles for the following four trials. In addition, single-pulse ES (1 Hz, square-wave pulse of alternating polarity, $0.3 \mathrm{~ms}$ duration, 12 or $15 \mathrm{~mA}$, four trials) was also applied to the same electrodes in order to record motor evoked potentials (MEPs) from EMG of the facial muscles (Ikeda et al., 2000; Kikuchi et al., 2012). 


\section{Results}

In both patients, mirth was elicited by high-frequency ES at a very restricted area in the

left basal temporal cortex [Fig. 1: a white circle (monopolar stimulation) in Patient 1

and black circles (bipolar stimulation) in Patient 2]. In Patient 1, high-frequency ES at

low intensity ( $5 \mathrm{~mA}, 5 \mathrm{sec}$ ) consistently caused lifting of the right side of the mouth,

followed by bilateral facial movements with mirth (Fig. 2). After ES was over, the

patient said, "I do not know why, but something amused me and I laughed.” In Patient 2,

high-frequency ES at low intensity and short duration (5 mA, $1 \mathrm{sec}$ ) produced mirth

without laughter. The stimulus intensity was gradually increased, and she eventually

developed laughter at high intensity and long duration (15 mA, 5 sec). Her introspection

was that "A familiar melody that I had heard in a television program in my childhood

came to mind; its tune sounded funny and amused me.” In both cases, mirth was elicited

only during high-frequency ES. In Patient 1, sham stimulation confirmed that the

feeling of mirth was actually induced by high-frequency ES (no specific feeling was

evoked by sham stimulation). These findings were reproducibly elicited by

high-frequency ES without afterdischarges (7/7 trials in Patient 1 and 14/14 trials in 
Patient 2).

In Patient 1, surface EMG recording during ES (50 Hz, 5 mA, 5 sec, four

trials) showed that the contralateral facial muscle contractions occurred at a mean of 3.3

sec (ranging from 2.8 to $3.7 \mathrm{sec}$ ) after the start of $50 \mathrm{~Hz}$ stimulation, and the onset of bilateral facial movements occurred at a mean of $0.3 \mathrm{sec}$ (ranging from 0.2 to $0.4 \mathrm{sec}$ )

after that of unilateral contractions. The onset of laughter in Patient 2 occurred $>5 \mathrm{sec}$

after stimulation as judged from video inspection (2 trials). In Patient 1, single-pulse ES

(1 Hz) was also applied to the electrode at which high-frequency ES caused mirth and

facial muscle contractions (white circle in Fig. 1). Single-pulse ES at the maximum

clinically safe intensity of $15 \mathrm{~mA}$ did not produce contralateral facial movements, MEPs, or mirth.

For clinical purposes, we evaluated language function in the basal temporal cortex, with the exception of the three (Patient 1) and six (Patient 2) electrodes where precise language mapping was difficult due to frequent afterdischarges. Stimulation of the electrodes that produced mirth had different results in the two patients. Mirth and laughter did not allow Patient 1 to perform language tests properly (white circle in Fig. 
1). In Patient 2, language impairment was reproducibly induced (15 mA, 5 sec, 4/4 trials,

black circles in Fig.1). In other parts of the left basal temporal cortex, six electrodes

resulted in language impairment (white and black diamonds), 11 resulted in no

impairment (small gray circles), and for nine electrodes the results were inconclusive

due to frequent afterdischarges (“x” marks). Electrodes associated with mirth were

located adjacent to the electrodes related to language function in both patients. In order

to clarify the relationship between mirth and language, the ratio of electrodes that

elicited language impairment was compared between electrodes that were associated

with mirth ("mirth electrode,” 3 electrodes) and those that were not (“no-mirth

electrode,” 17 electrodes) (electrodes with inconclusive results due to afterdischarges

were excluded). The ratio of electrodes eliciting language impairment was higher for the

mirth electrode $(2 / 3=0.66)$ than the no-mirth electrodes $(6 / 17=0.32)$.

\section{Discussion}

\subsection{Mirth and language}

The neural basis of mirth has been explored by studying humor, classically via brain

lesions and more recently with fMRI. Humor can be divided into cognitive and affective 
processing (Gardner et al., 1975). Cognitive processing, i.e., humor detection, is formed in two stages: 1) a perception of incongruity between the expectation and "punch line”, and 2) resolution of the incongruity (Suls, 1972). A series of fMRI studies of humor indicated that cognitive processing has phonological and semantic components. The phonological component was associated with activation in the left lateral inferior frontal gyrus (Goel \& Dolan, 2001), which was a consistent finding in the previous fMRI studies (Amir et al., 2013; Mobbs et al., 2003; Moran et al., 2004; Watson et al., 2007). The semantic component of humor processing was associated with activation in the temporo-occipital and temporo-parietal junctions; bilateral activation was observed in tasks involving humor interpretation (Amir et al., 2013), listening to different types of jokes (Goel \& Dolan, 2001), and processing of sight gag-related humor (Watson et al., 2007), while some studies using funny cartoons (Mobbs et al., 2003) or verbal gags (Watson et al., 2007) reported the dominance in the left hemisphere. Although the anterior part of the basal temporal cortex is regarded as one of the core regions in the semantic network (Binney, Embleton, Jefferies, Parker, \& Ralph, 2010; Mion et al., 2010; Shimotake et al., 2014), only one of these fMRI studies reported activation of this 
region. This is mainly due to a variety of technical factors including magnetic field inhomogeneities (for standard gradient echo planar imaging) and limited field of view (Devlin et al., 2000; Visser, Embleton, Jefferies, Parker, \& Ralph, 2010). Moran et al. (2004) suggested that semantic processing brings stored expectations, and that phonological processing then resolves the incongruities between these expectations and punch lines, supporting the incongruity-resolution theory of humor.

Affective processing, i.e., humor appreciation, is thought to be associated with the mesolimbic dopaminergic reward system. Activation was reported in the bilateral ventral striatum, nuclei accumbens, ventral tegmenta, hypothalami, and amygdalae during humor appreciation of funny cartoons (Mobbs et al., 2003), as well as in the right medial prefrontal cortex while listening to different types of jokes (Goel \& Dolan, 2001). These reward-related regions were also activated in other fMRI studies to varying degrees: the left amygdala, left middle prefrontal cortex and bilateral ventral striatum (Amir et al., 2013), the bilateral amygdalae and insulae (Moran et al., 2004), and the left amygdala and nucleus accumbens (Watson et al., 2007). Amir et al. (2013) hypothesized that the regions associated with humor detection trigger these 
reward-related regions, producing the hedonic feeling that accompanies humor.

ES has been the gold standard for mapping brain function for functional neurosurgery, even though it is an invasive technique. Only a handful of studies have applied this technique to elucidate the cortical network associated with mirth and laughter. Laughter with mirth was produced during ES of the left basal temporal area (anterior parahippocampal and fusiform gyri) (Arroyo et al., 1993; Satow et al., 2003), the left inferior frontal gyrus (pars opercularis) (Fernandez-Baca Vaca, Lüders, Basha, \& Miller, 2011), and the left medial frontal lobe (the rostral part of the supplementary motor area [SMA]) (Fried, Wilson, MacDonald, \& Behnke, 1998; Krolak-Salmon et al., 2006). Laughter without mirth was elicited by ES of the left or right cingulate gyrus (Arroyo et al., 1993; Sperli, Spinelli, Pollo, \& Seeck, 2006), the right medial frontal lobe (the rostral part of SMA), and the left lateral dorsal premotor cortex (Schmitt, Janszky, Woermann, Tuxhorn, \& Ebner, 2006). In summary, in our two cases and in several previously reported cases with definite language dominance (by the Wada test) or handedness (Arroyo et al., 1993; Fernandez-Baca Vaca et al., 2011; Krolak-Salmon et al., 2006; Satow et al., 2003), mirth was elicited by ES only at the frontal and 
temporal cortices of the dominant left hemisphere. Interestingly, when ES was

performed for clinical functional cortical mapping, all of the electrodes producing mirth were located adjacent to language-related areas: the left SMA (Fried et al. 1998), Broca’s area (Fernandez-Baca Vaca et al., 2011), and the left BTLA (Arroyo et al., 1993). Moreover, in our cases the ratio of electrodes eliciting language impairment was higher for the mirth electrodes than in no-mirth electrodes, suggesting an association between mirth and language function. Since the BTLA is actively involved in semantic processing (Shimotake et al., 2014; Usui et al., 2003), this semantic/language area was likely involved in the semantic aspect of humor detection in our cases.

The stimulation studies cited above demonstrated that the left hemisphere, which is dominant for language, is more related to mirth, but the findings should be interpreted carefully, for two reasons. First, since the invasive ES mirth studies were performed more frequently in the hemisphere dominant for language to identify brain functions, we currently do not have enough ES findings regarding mirth and laughter in the hemisphere non-dominant for language, especially in the cortical regions homologous to those producing mirth, with or without laughter, in the hemisphere 
dominant for language. Second, humor fMRI studies in healthy right-handed subjects

showed that bilateral temporal cortices were involved in semantic processing while the left hemisphere was activated during language perception and production. Only a few brain stimulation studies reported that stimulation of the nucleus accumbens (Okun et al., 2004) or subthalamic nucleus (Krack et al., 2001) produced laughter and mirth. Further ES studies are warranted for comprehensive mapping of the cortical and subcortical regions associated with mirth and laughter by accumulating the ES findings of the non-dominant hemisphere and of subcortical structures.

\subsection{A suggested mechanism for mirth and facial movements}

To the best of our knowledge, this is the first report using high-frequency ES with EMG recordings of the facial muscles to demonstrate concomitant contralateral facial movements with mirth. In a tracer study in rhesus monkeys, the primary motor, premotor, and caudal cingulate cortices projected primarily to the contralateral lateral subnucleus of the facial nucleus, while the supplementary motor and rostral cingulate cortices projected bilaterally to the dorsal and intermediate subnuclei (Morecraft, Louie, Herrick, \& Stilwell-Morecraft, 2001). Two independent neuronal pathways are 
proposed for the expression of laughter (Wild, Rodden, Grodd, \& Ruch, 2003). The first, the volitional pathway, runs from motor cortices to the ventral brainstem through the pyramidal tract. The second, the emotional pathway, involves the limbic system and leads to the dorsal brainstem. However, it is still unclear which pathways are involved in the association between facial movements and mirth. In a human lesion study that investigated a stroke of the primary facial motor cortex, involuntary facial movements during mirth were preserved and were hypothesized to arise from the cingulate cortex (Kappos \& Mehling, 2010). Contralesional mimetic facial palsy, namely, unilateral emotional facial paresis, was demonstrated in patients with mesial temporal lobe epilepsy with HS (Hopf et al., 1992), the latter of which is diagnosed on the basis of neuronal cell loss and gliosis of mesial temporal structures, including the amygdala (Wieser, 2004). These studies suggest that the limbic system, especially the anterior cingulate cortex and mesial temporal structures such as the amygdala, is involved in the association between facial movements and mirth.

In Patient 1, high-frequency ES (5 mA) evoked contraction of contralateral facial muscles, and its onset time was shorter than that of laughter with mirth in both 
patients, while single-pulse ES (15 mA) produced neither facial movements nor MEPs.

This discrepancy implies that the basal temporal cortex is not connected

monosynaptically with a motor pathway such as the corticobulbar tract, but rather

multisynaptically with the contralateral facial motor nucleus through the limbic system.

Indeed, the corresponding region in rhesus monkeys has connections with the amygdala and the perirhinal cortex (Schmahmann \& Pandya, 2006). In Patient 1, the impulse produced by ES at the anterior basal temporal area was most likely transmitted first to the normal amygdala and subsequently to the limbic system, leading first to contralateral facial contraction and then to bilateral facial movements.

In Patient 1, who had a normal hippocampus, high-frequency ES most likely produced contralateral facial movements through the normal emotional pathway. On the other hand, in Patient 2, who had HS and contralesional mimetic facial palsy, the normal emotional pathway was disturbed, and therefore high-frequency ES presumably produced mirth, followed by facial movements through the volitional pathway. In other words, we likely observed two sides of the same coin regarding the emotional pathway: contralateral facial contraction by $50 \mathrm{~Hz}$ stimulation through the normal mesial 
temporal structure in Patient 1, and contralateral emotional facial paresis because of the pathological mesial temporal structure (HS) in Patient 2. We therefore postulate that mesial temporal structures play an important role in the onset of facial movements associated with mirth. We also postulate that these facial movements have contralateral dominance, as was shown to be the case with the second somatosensory area and supplementary motor area (Babiloni et al., 2003; Mima et al., 1997). One ES study showed that ES of the right anterior cingulate cortex produced contralateral facial movements followed by bilateral facial movements, without mirth (Sperli et al., 2006). In a deep brain stimulation study, stimulation of the nucleus accumbens produced contralateral facial movements with euphoria (Okun et al., 2004). These studies further support our hypothesis.

In summary, the present direct stimulation study showed that 1) mirth had a close relationship with language function, 2) intact mesial temporal structures actively engaged at the beginning of facial movements associated with mirth, and 3) these emotional facial movements had contralateral dominance. 


\section{ACKNOWLEDGMENTS}

We are indebted to Dr. Keiko Usui for providing the patient data. This work was partly

supported by Grants-in-Aid for Scientific Research (B) 26282218 (RM), (C) 24592159

(TK), and Exploratory Research 26560465 (RM) from the Ministry of Education,

Culture, Sports, Science and Technology of Japan. Department of Epilepsy, Movement

Disorders and Physiology, Kyoto University Graduate School of Medicine is an

endowment department, supported with grants by GlaxoSmithKline K.K., NIHON

KOHDEN CORPORATION, Otsuka Pharmaceutical Co., and UCB Japan Co., Ltd. 


\section{REFERENCES}

Amir, O., Biederman, I., Wang, Z., \& Xu, X. (2013). Ha Ha! Versus Aha! A Direct

Comparison of Humor to Nonhumorous Insight for Determining the Neural

Correlates of Mirth. Cereb Cortex.

Arroyo, S., Lesser, R. P., Gordon, B., Uematsu, S., Hart, J., Schwerdt, P., et al. (1993).

Mirth, laughter and gelastic seizures. Brain, 116 ( Pt 4), 757-780.

Babiloni, C., Carducci, F., Del Gratta, C., Demartin, M., Romani, G. L., Babiloni, F., et al. (2003). Hemispherical asymmetry in human SMA during voluntary simple unilateral movements. An fMRI study. Cortex, 39, 293-305.

Binney, R. J., Embleton, K. V., Jefferies, E., Parker, G. J., \& Ralph, M. A. (2010). The ventral and inferolateral aspects of the anterior temporal lobe are crucial in semantic memory: evidence from a novel direct comparison of distortion-corrected fMRI, rTMS, and semantic dementia. Cereb Cortex, 20, 2728-2738.

Devlin, J. T., Russell, R. P., Davis, M. H., Price, C. J., Wilson, J., Moss, H. E., et al. (2000). Susceptibility-induced loss of signal: comparing PET and fMRI on a 
semantic task. NeuroImage, 11, 589-600.

Fernandez-Baca Vaca, G., Lüders, H. O., Basha, M. M., \& Miller, J. P. (2011). Mirth

and laughter elicited during brain stimulation. Epileptic Disord, 13, 435-440.

Fried, I., Wilson, C. L., MacDonald, K. A., \& Behnke, E. J. (1998). Electric current stimulates laughter. Nature, 391, 650.

Gardner, H., Ling, P. K., Flamm, L., \& Silverman, J. (1975). Comprehension and appreciation of humorous material following brain damage. Brain, 98, 399-412.

Goel, V., \& Dolan, R. J. (2001). The functional anatomy of humor: segregating cognitive and affective components. Nat Neurosci, 4, 237-238.

Hopf, H. C., Muller-Forell, W., \& Hopf, N. J. (1992). Localization of emotional and volitional facial paresis. Neurology, 42, 1918-1923.

Ikeda, A., Ohara, S., Matsumoto, R., Kunieda, T., Nagamine, T., Miyamoto, S., et al. (2000). Role of primary sensorimotor cortices in generating inhibitory motor response in humans. Brain, 123 ( Pt 8), 1710-1721.

Kappos, L., \& Mehling, M. (2010). Images in clinical medicine. Dissociation of voluntary and emotional innervation after stroke. $N$ Engl J Med, 363, e25. 
Kikuchi, T., Matsumoto, R., Mikuni, N., Yokoyama, Y., Matsumoto, A., Ikeda, A., et al. (2012). Asymmetric bilateral effect of the supplementary motor area proper in the human motor system. Clin Neurophysiol, 123, 324-334.

Krack, P., Kumar, R., Ardouin, C., Dowsey, P. L., McVicker, J. M., Benabid, A. L., et al. (2001). Mirthful laughter induced by subthalamic nucleus stimulation. Mov Disord, 16, 867-875.

Krolak-Salmon, P., Hénaff, M. A., Vighetto, A., Bauchet, F., Bertrand, O., Mauguière, F., et al. (2006). Experiencing and detecting happiness in humans: the role of the supplementary motor area. Ann Neurol, 59, 196-199.

Matsumoto, R., Imamura, H., Inouchi, M., Nakagawa, T., Yokoyama, Y., Matsuhashi, M., et al. (2011). Left anterior temporal cortex actively engages in speech perception: A direct cortical stimulation study. Neuropsychologia, 49, $1350-1354$.

Mikuni, N., Miyamoto, S., Ikeda, A., Satow, T., Taki, J., Takahashi, J., et al. (2006). Subtemporal hippocampectomy preserving the basal temporal language area for intractable mesial temporal lobe epilepsy: preliminary results. Epilepsia, 47, 
1347-1353.

Mima, T., Ikeda, A., Nagamine, T., Yazawa, S., Kunieda, T., Mikuni, N., et al. (1997).

Human second somatosensory area: subdural and magnetoencephalographic

recording of somatosensory evoked responses. J Neurol Neurosurg Psychiatry,

63, 501-505.

Mion, M., Patterson, K., Acosta-Cabronero, J., Pengas, G., Izquierdo-Garcia, D., Hong, Y. T., et al. (2010). What the left and right anterior fusiform gyri tell us about semantic memory. Brain, 133, 3256-3268.

Mobbs, D., Greicius, M. D., Abdel-Azim, E., Menon, V., \& Reiss, A. L. (2003). Humor modulates the mesolimbic reward centers. Neuron, 40, 1041-1048.

Moran, J. M., Wig, G. S., Adams, R. B., Jr., Janata, P., \& Kelley, W. M. (2004). Neural correlates of humor detection and appreciation. NeuroImage, 21, 1055-1060.

Morecraft, R. J., Louie, J. L., Herrick, J. L., \& Stilwell-Morecraft, K. S. (2001). Cortical innervation of the facial nucleus in the non-human primate: a new interpretation of the effects of stroke and related subtotal brain trauma on the muscles of facial expression. Brain, 124, 176-208. 
Okun, M. S., Bowers, D., Springer, U., Shapira, N. A., Malone, D., Rezai, A. R., et al. (2004). What's in a "smile?" Intra-operative observations of contralateral smiles induced by deep brain stimulation. Neurocase, 10, 271-279.

Satow, T., Usui, K., Matsuhashi, M., Yamamoto, J., Begum, T., Shibasaki, H., et al. (2003). Mirth and laughter arising from human temporal cortex. J Neurol Neurosurg Psychiatry, 74, 1004-1005.

Schmahmann, J. D., \& Pandya, D. N. (2006). Fiber Pathways of the Brain: Oxford Univ. Press.

Schmitt, J. J., Janszky, J., Woermann, F., Tuxhorn, I., \& Ebner, A. (2006). Laughter and the mesial and lateral premotor cortex. Epilepsy Behav, 8, 773-775.

Shimotake, A., Matsumoto, R., Ueno, T., Kunieda, T., Saito, S., Hoffman, P., et al. (2014). Direct exploration of the role of the ventral anterior temporal lobe in semantic memory: Cortical stimulation and local field potential evidence from subdural grid electrodes. Cereb Cortex, http://dx.doi.org/10.1093/cercor/bhu262. Sperli, F., Spinelli, L., Pollo, C., \& Seeck, M. (2006). Contralateral smile and laughter, but no mirth, induced by electrical stimulation of the cingulate cortex. Epilepsia, 
47, 440-443.

Suls, J. (1972). A two-stage model for the appreciation of jokes and cartoons:an

information-processing analysis. The Psychology of Humor: Theoretical

Perspectives and Empirical Issues. New York: Academic Press.

Takayama, M., Miyamoto, S., Ikeda, A., Mikuni, N., Takahashi, J. B., Usui, K., et al.

(2004). Intracarotid propofol test for speech and memory dominance in man.

Neurology, 63, 510-515.

Usui, K., Ikeda, A., Takayama, M., Matsuhashi, M., Yamamoto, J., Satoh, T., et al.

(2003). Conversion of semantic information into phonological representation: a

function in left posterior basal temporal area. Brain, 126, 632-641.

Visser, M., Embleton, K. V., Jefferies, E., Parker, G. J., \& Ralph, M. A. (2010). The inferior, anterior temporal lobes and semantic memory clarified: novel evidence from distortion-corrected fMRI. Neuropsychologia, 48, 1689-1696.

Watson, K. K., Matthews, B. J., \& Allman, J. M. (2007). Brain activation during sight gags and language-dependent humor. Cereb Cortex, 17, 314-324.

Wieser, H. G. (2004). ILAE Commission Report. Mesial temporal lobe epilepsy with 
hippocampal sclerosis. Epilepsia, 45, 695-714.

Wild, B., Rodden, F. A., Grodd, W., \& Ruch, W. (2003). Neural correlates of laughter and humour. Brain, 126, 2121-2138. 


\section{Figure legends}

\section{Fig. 1}

The results of high-frequency electrical cortical stimulation are shown in the Montreal Neurological Institute (MNI) standard space. "Mirth” (circles) and “language” (diamonds) electrodes are shown in white and black colors for Patients 1 and 2, respectively. Gray circles denote electrodes with no function, and " $x$ " marks those with inconclusive results due to frequent afterdischarges. Only the electrodes placed in the basal temporal lobe are shown. Note that mirth was elicited at or adjacent to the electrode associated with language impairment. The brainstem and cerebellum are not shown for clarity. $\mathrm{R}$ = right side.

Fig. 2

Unilateral facial movement preceding mirth in Patient 1. Facial expressions and rectified electromyograms of bilateral levator labii superior muscles are shown; results of four trials ( $50 \mathrm{~Hz}, 5 \mathrm{~mA}, 5 \mathrm{sec}$ ) are superimposed using black and three shades of gray. High-frequency electrical stimulation at the "mirth” electrode (white circle in Fig. 
1) produced lift of the right mouth via unilateral contraction of the right levator labii superior muscle (middle photo), which was followed by mirth with bilateral facial movements (right photo). 
(98. 京都大学

Fig. 1

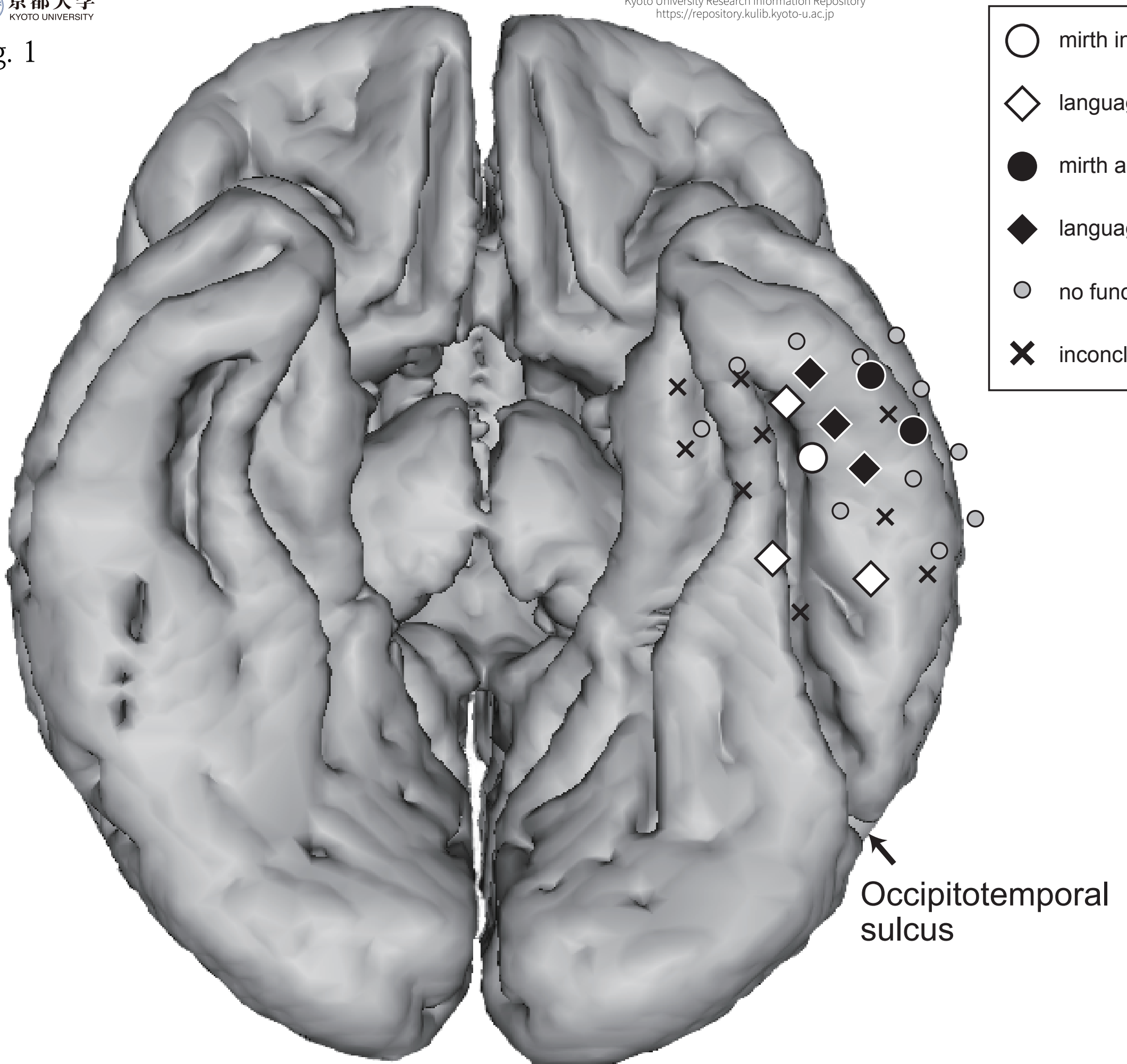

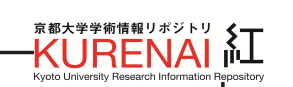

language impairment in Patient 1

mirth and language impairment in Patient 2

anguage impairment in Patient 2

function

conclusive due to afterdischarges

\begin{tabular}{|cccc|}
\hline \multicolumn{4}{c}{ MNI coordinate } \\
& $x$ & $y$ & $z$ \\
\hline 0 & -44 & -20 & -38 \\
0 & -52 & -8 & -42 \\
& -56 & -16 & -40 \\
\hline
\end{tabular}


Right levator labii superior muscle

Left levator labii superior muscle

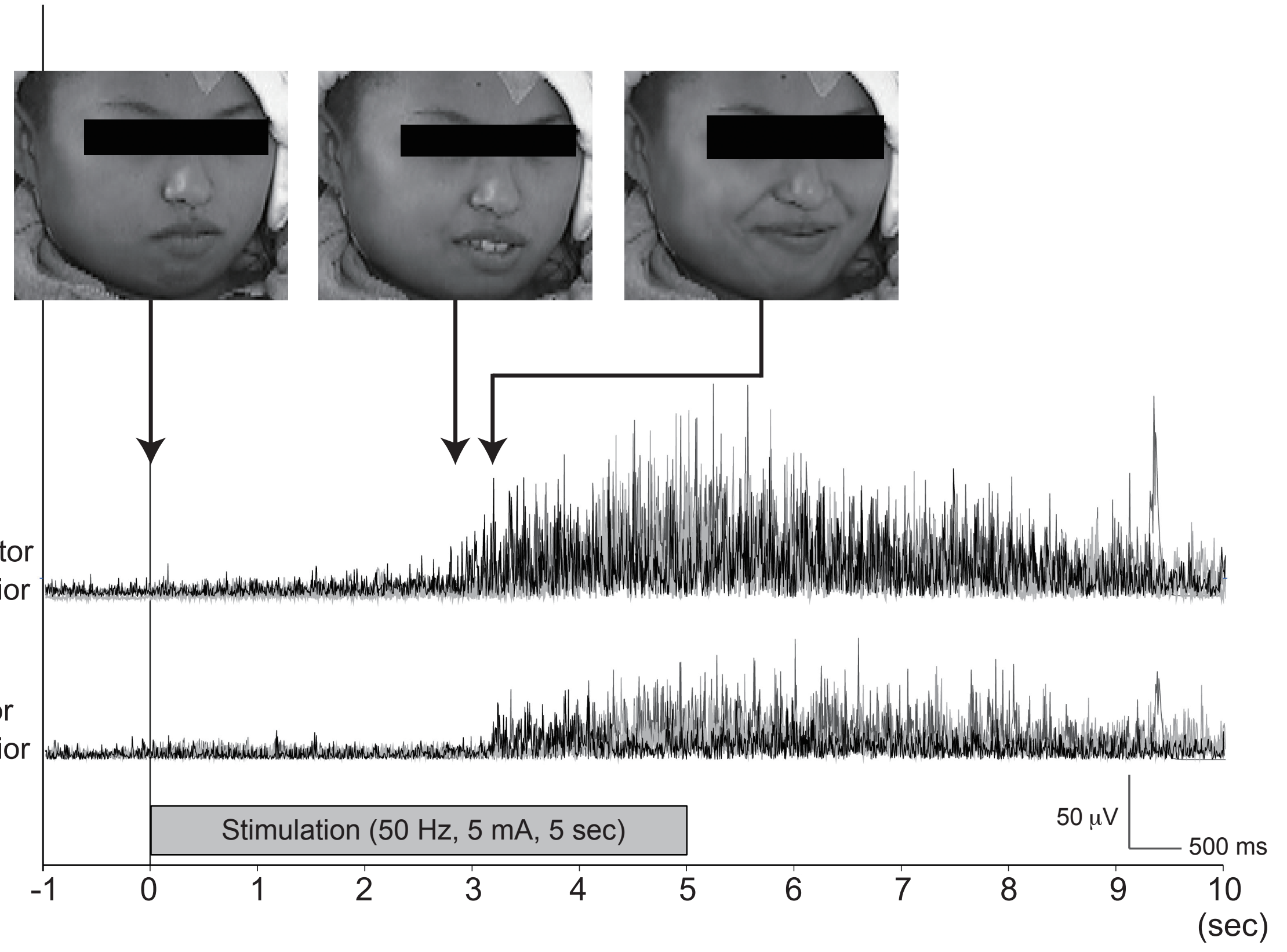

